\title{
7 Kein Zurückschrecken vor der Psychose. Jacques Lacan zum Wahn
}

\author{
Ulrike Kadi und August Ruhs
}

Noch vor zwanzig Jahren war es durchaus üblich, darauf zu verweisen (Apollon 1991), dass Psychoanalyse für eine Behandlung von Psychosen nicht viel beizutragen habe. Salopp ausgedrückt hieß es diesbezüglich in Psychiaterkreisen Frankreichs: „La psychanalyse pour les névroses, les psychoses sont autre chose" (Die Psychoanalyse für die Neurosen, die Psychosen sind etwas anderes). Im Hinblick auf den französischen Psychoanalytiker Jacques Lacan muss das zunächst erstaunen, war doch für ihn im Unterschied zu Freud die Psychose und nicht die Neurose der Ausgangspunkt seiner psychoanalytischen Konzepte. Darüber hinaus bildete die psychotische Struktur über vier Jahrzehnte hinweg einen Fokus seiner Überlegungen. Hinsichtlich der Therapiefähigkeit von Psychotikern war Lacan allerdings wie Freud der Ansicht, dass das psychoanalytische Standardverfahren kontraindiziert wäre, dass aber ein abgeändertes psychoanalytisches Vorgehen durchaus gewinnbringend sei (vgl. Ruhs 2001).

Die Vielschichtigkeit der Vorschläge Lacans in den unterschiedlichen Phasen seiner theoretischen Annäherung an die Psychosen wurde lange Zeit wenig beachtet. Das zeigt sich etwa daran (Brousse 2009), dass sein drittes Seminar über Die Psychosen (Lacan 1997) und die Zusammenfassung dieses Seminars (Lacan 1975) in den Vordergrund gerückt und seine Konzepte aus den 1930er und aus den 1970er-Jahren vernachlässigt wurden (vgl. als Beispiel Waelhens u. Ver Ecke 2001).

Bezüglich der Rezeption Lacans, seines Beitrags zu einer Theorie und Behandlung von psychotischen Zuständen sowie zur Frage der Psychoanalyse von Psychosen hat sich nicht nur im U.S.-amerikanischen, sondern auch im frankophonen Diskussionsbereich der Psychoanalyse inzwischen viel verändert. Die Diagnose einer Psychose, die vorher vielfach deshalb erfolgen sollte, um die Indikation einer Psychoanalyse per exclusionem einer Psychose zu stellen, hat damit neuen Wert bekommen. Sie dient nicht mehr dazu, eine psychoanalytische Behandlung vor allem neurotischen Strukturen vorzubehalten, sondern sie erfolgt, damit das psychoanalytische Vorgehen in der Behandlung auch von Subjekten mit psychotischer Struktur strukturkonform erfolgen kann. 
Auch wenn Lacan in den 1970er-Jahren zur Auffassung kommt, dass Analytiker vor einer Psychose nicht zurückschrecken brauchen (Lacan 1977), gilt es einer seiner früheren Schriften zufolge dennoch, eine bestimmte Frage vor jeder Behandlung einer Psychose anzugehen (Lacan 1975). Diese Ansicht vertrat er in den 1950er-Jahren (Lacan 1997). Und auch zwanzig Jahre später rückte er nicht von dieser Vorgabe ab: Vor jeder Behandlung ist nach der Existenz des Subjekts bzw. nach der Struktur des/derjenigen zu fragen, der/die sich einer Kur unterziehen möchte (vgl. dazu auch Fink 1999). Diese Empfehlung mag verwundern, ist doch eine Ablehnung des Diagnostizierens noch vor Beginn einer Behandlung aus ethischen wie epistemologischen Gründen gegenwärtig unter PsychoanalytikerInnen nichts Ungewöhnliches (Ruhs 2005 u. Brousse 2009). Hier soll der mehrschichtige Kontext der Empfehlung Lacans dargestellt werden. Im Anschluss daran werden seine verschiedenen Theorien zum Wahn unter vier Gesichtspunkten betrachtet. Jene Momente in den Wahnbildungen von Patient 1 (Stompe 2010) und Patientin 2 (s. Anhang), die aus einer Lacanianischen Perspektive erhellt werden können, sind in die Darstellung eingeflochten.

\subsection{Vor jeder Behandlung}

Ein Wahn ist ein Symptom. Als solches gilt er gleichermaßen in einer psychiatrischen wie in einer nicht weiter spezifizierten psychoanalytischen Betrachtung. Trotz dieser oberflächlichen Ähnlichkeit finden sich eine Reihe von Unterschieden zwischen der psychoanalytischen und der psychiatrischen Weise, einen Wahn zu diagnostizieren. Im Rahmen einer psychiatrischen Diagnosefindung wird eine Auflistung von symptomhaft aufgefassten Phänomenen unter Einschluss von Zeitkriterien dazu verwendet, um Syndrome zu beschreiben, die einer Störung zugeordnet werden können. Der Wahn fungiert dabei insofern als Symptom, als sein Vorkommen pathognomonisch für eine Diagnose ist.

Am Beginn einer psychoanalytischen Herangehensweise steht jedoch die Unterscheidung zwischen oberflächlichen Anzeichen, Symptomen und einer gegenüber dieser Oberfläche weniger leicht erkennbaren Struktur eines Subjekts, wobei über das Gewicht der Symptome für eine individuelle Strukturdiagnose zunächst nichts gesagt werden kann. Denn die oberflächlichen Anzeichen und Symptome können aus mehreren Gründen kein klares und einheitliches Kriterium für ein psychoanalytisches Verständnis der Struktur bilden. Sie können nicht nur in Opposition zur Struktur geraten, sondern geradezu eingesetzt sein, um eine Struktur zu verdecken. Phobien, Zwänge oder Konversionssymptome etwa wären daher aus psychoanalytischer Sicht mit der Diagnose einer Psychose gut vereinbar (Cremniter u. Maleval 1989). Lacan geht 1956 sogar so weit, zu behaupten, dass nichts einer neurotischen Struktur ähnlicher sei als jener Zustand, den er Präpsychose nennt (Lacan 1997). Neben einer den Symptomen immanenten Tendenz zur Verdeckung rückt in der psychoanalytischen Annäherung zudem die auch für eine psychiatrische Diagnose bekannte Tatsache in den Vordergrund, dass dieselben Symptome als Träger unterschiedlicher Bedeutung funktionieren. Diese Polysemie des Symptoms macht es unmöglich, vom Symptom direkt auf eine Struktur zu schließen. Der Wahn, ein König zu sein (Kadi 2012a), kann Ausdruck eines Wunsches nach einer höheren Abstammung sein und wäre hierin durchaus als ein neurotisches Symptom zu verstehen (Freud 1909). Ein Subjekt kann aber durch dieselbe unverrückbare Gewissheit, ein König zu sein, auch zum Ausdruck bringen, dass es keine Königin ist. Derselbe Wahn würde dabei vom Subjekt dazu verwendet werden, sich eine Geschlechtsidentität angesichts einer sexuellen Ambivalenz zu verschaffen, was weniger an ein neurotisches als an ein psychotisches Sym- 
ptom denken ließe. Eine solche Mehrdeutigkeit findet sich auch im Bericht der Patientin 2 (Frau S.). Jean Paul Sartre zu sein, kann heißen, gut schreiben zu können eine durchaus noch im Rahmen des Neurotischen vorstellbare Überwindung der eigenen Grenzen. Sartre zu sein, kann aber auch bedeuten, ein Mann zu sein, worin sich Frau S. eine Gewissheit über ihr Geschlecht vermittelt, die ihr während dieser Phase eines psychotischen Erlebens abhandengekommen ist.

In dieser Funktion des Wahns für die Patientin deutet sich eine andere Möglichkeit als jene an, Symptome als polysemische Anzeichen täuschender oder nicht täuschender Art anzusehen. Dieser andere Blick auf Symptome war psychoanalytisch von Anfang an eingeführt und wurde für Lacan in seinen späten Arbeiten maßgeblich: Symptome sind von Freud unter anderem als Kompromissbildungen (Freud 1922), im Fall des Wahns als Selbstheilungsversuche eines Subjekts angesehen worden (Freud 1911, 1917), eine Annahme, die Freud, vom Symptom ausgehend, schließlich sogar auf ganze Erkrankungsbilder ausdehnt (Freud 1937). Lacan macht sich diese Betrachtungsweise in seiner späten Theorie des von ihm herausgearbeiteten „Sinthoms“ zunutze, um das kreative Moment von Symptomen in den Vordergrund zu rücken. Symptome gelten ihm dabei in besonderer Weise nicht nur als Krankheitszeichen, sondern als mehr oder minder geglückte Momente der Bewältigung mit strukturmodifizierender Potenz. Daher ist für eine verlässliche Diagnose einer psychotischen Struktur nach Lacan beides nötig: Der Blick auf die klinischen Zeichen und der Blick auf den Ort einer möglicherweise geglückten Kompensation (Cremniter u. Maleval 1989).

Für die Behandlung ist es wichtig, sich ein Bild darüber zu machen, ob im Rahmen einer Psychoanalyse bei einem bestimmten Subjekt eine psychotische Dekompensation zu erwarten ist. Freud berichtet diesbezüglich vom Abbruch einer Kur, da die Patientin hospitalisiert werden musste (Freud 1894). Lacan hat wie Freud immer wieder darauf hingewiesen, dass eine Behandlung auf der Couch psychotische Krisen befördern kann, die teilweise bis in den Selbstmord führen (Cremniter u. Maleval 1989). Lacan zufolge wäre die Nichtbeachtung einer psychotischen Struktur im Rahmen einer klassischen Kur ähnlich stupid wie ein Rudern, wenn ein Schiff auf Sand gelaufen ist (Lacan 1975).

Doch wie lässt sich eine psychotische Exazerbation voraussagen, wenn Symptome falsche Fährten legen oder mehrdeutig sind? Woran soll sich ein prospektiver Analytiker orientieren, wenn er die Zeichen, auf die sich andere verlassen, nicht als Anzeichen ansehen kann? Die beiden Fallberichte, die hier zugrunde zu legen sind, verdecken diese spezielle Situation vor Beginn jeder Psychoanalyse. Denn beide legen den Fokus auf psychotische Krisen, was das Diagnostizieren erleichtert. Vor Beginn einer Psychoanalyse ist aber auch bei jenen Subjekten nach einer Orientierung zu suchen, die anamnestisch derlei Krisen nicht aufweisen. Insbesondere wenn solche Subjekte später Momente einer Psychose zeigen, ist zu fragen, unter welcher Kategorie sie nachträglich - noch vor jedem Ausbruch einer Psychose - zu klassifizieren gewesen wären. Lacan hat dafür in seinen Überlegungen zu Schreber den bereits erwähnten Terminus „Präpsychose“ gewählt (Lacan 1997), ein Terminus, den er wenige Jahre später wieder fallen lässt (Cremniter u. Maleval 1989). Auch der Begriff der „blanden Psychose“, vor allem aber der von Jacques-Alain Miller in den späten 199oerJahren geprägte Begriff der „gewöhnlichen Psychose“/,Psychose ordinaire“ (Miller 2005 u. Maleval 2003) sind Versuche, Inklinationen zur Psychose im Vorfeld einer psychotischen Entwicklung zu fassen. 
Alle diese Vorbemerkungen sowohl zu den Fallstricken als auch zum Für und Wider einer Diagnose sind - auch wenn sie zum Verständnis der beiden Fallgeschichten zunächst nur wenig beizutragen scheinen - integraler Bestandteil jeglicher an Lacan orientierten Herangehensweise an psychopathologische Bildungen. Und es kommt ein weiterer Aspekt hinzu: Es wäre kurzsichtig zu glauben, eine Diagnose ließe sich auf einen Prozess reduzieren, in dessen Rahmen ein prospektiver Analysant (Lacan betont mit dem Partizip Präsens „Analysant“ die aktive Funktion des In-AnalyseSeins.) einen Namen für seinen Zustand erfährt. Eine Diagnose umfasst implizit und meist wenig reflektiert auch den Diagnostiker, sei er Psychiater oder Analytiker, als denjenigen, der sich selbst im Rahmen des diagnostischen Prozesses als Subjekt zu bestimmen versucht. Jemanden anderen als nicht-normal, als neurotisch, psychotisch oder pervers zu deklarieren, enthält eine Mitteilung auch darüber, in welche Position sich derjenige, der diagnostiziert, selbst rückt. Sehr häufig wird dabei von einer unausgesprochenen Normalitätsannahme für den Diagnostiker ausgegangen eine Voraussetzung, die, wie sich unten zeigen wird, aus Sicht der späteren Seminare Lacans vor allem als eine Selbsttäuschung anzusehen ist. Dies lässt sich etwa an Lacans Ansicht ablesen, dass es kein Privileg von James Joyce sei, verrückt zu sein (Lacan 2005).

Die unausgesprochene Normalitätsannahme für den Diagnostiker stützt sich nämlich auf ein Modell der Psychose und mit ihr des Wahns, in dem beide als radikal unterschieden und unterscheidbar gegenüber dem Normalen gedacht werden. Lacan war eine solche Sicht schon früh fremd, was sich unter anderem in seiner Behauptung zeigt: Ein Narr, der glaubt ein König zu sein, ist nicht verrückter als ein König, der glaubt, ein König zu sein (Lacan 1980). Worauf hier verwiesen wird, ist die Tatsache, dass sich das Sein nicht in eine Funktion bzw. einen Signifikanten verlegen lässt, was für Narr und König gleichermaßen gilt. Während Lacan früh festhält, dass das Phänomen des Wahns, hierin den Kunstwerken vergleichbar, nur von denjenigen verstanden werden könne, die sich von einem naiven Realismus des Gegenstandes gelöst hätten (Lacan 1933), behauptet er fast drei Jahrzehnte später, kein Psychiater und kein Psychoanalytiker verfüge über ein durch und durch adäquates Verhältnis zur Realität (Lacan 1975). Das lässt sich so verstehen, dass auch in sogenannten normalen Überzeugungen Züge enthalten sind, die dem Wahn in seiner Struktur gleichen. Welche Züge hat Lacan dabei vor allem im Auge? Von den drei Jaspers'schen Kriterien des Wahns ist es die subjektive Gewissheit, welche Lacan zufolge sowohl dem Wahn wie der normalen Überzeugung innewohnt. In Lacans Terminologie ausgedrückt, sind dazu fixe Verbindungen zwischen Signifikant, dem sprachlichen Ausdruck, und dem Signifikat, dem mit dem Ausdruck Gemeinten, nötig. Lacan nennt sie in Anlehnung an Verbindungen zwischen Ober- und Unterseite im Inneren einer Matratze Stepppunkte (les points de capiton). Sie kommen nicht nur bei Psychosen vor, sondern sind für jeden Prozess einer sprachlichen Verständigung notwendig und treten daher in beschränktem Ausmaß in sämtlichen Sprachgemeinschaften als ein Moment der Verankerung in dem für Sprache allgemein kennzeichnenden permanenten Gleiten zwischen Signifikant und Signifikat auf (Lacan 1997). Als Beispiel sei hier angeführt, dass der Signifikant „Sartre“ für die meisten von uns der Name eines französischen Philosophen ist und dass wir beim Lesen der Fallvignette nicht nachdenken, ob Jean-Paul Sartre vielleicht ein Filmstar oder ein Hausbesorger ist. Das Signifikat des existenzialistischen Philosophen ist einigermaßen fest mit dem Signifikanten Sartre verbunden. 
Lacan geht zunächst von einer grundsätzlichen Unterscheidungsmöglichkeit von Wahn und normaler Erfassung der Realität aus, auch wenn er eine Strukturähnlichkeit zwischen beiden in Form von (in unterschiedlichem Ausmaß bestehenden) fixen Verbindungen zwischen Signifikant und Signifikat aufzeigt. In seinen späteren Seminaren relativiert Lacan seinen früheren Ansatz: Zwischen einer vom Wahn geprägten Erfassung der Realität und einer sogenannten normalen Realitätserfassung kann er nun vor dem Hintergrund seiner Theorie der Geschlechter nur noch ein Kontinuum sehen. Diese Veränderung seiner Auffassung des Wahns steht in engem Zusammenhang mit den bereits erwähnten Änderungen seiner Theorie, insbesondere den Status des „Namens-des-Vaters“ betreffend, welcher Dreh- und Angelpunkt sowohl seiner gesamten früheren psychoanalytischen Auffassung über das Subjekt wie auch eine zentrale Kategorie seines Wahnverständnisses war. Daher sind hier nun zunächst jene Kategorien darzustellen, mit denen Lacan sich dem Wahn nähert. Sie erweisen sich als eingebettet in ein Verständnis des Subjekts, das seinerseits seit Lacans früher Arbeit über das Spiegelstadium (Lacan 1973) eng mit dem Wahn verbunden ist (Kadi 1999).

Anders als die heute übliche psychiatrische Diagnostik umfasst eine psychoanalytische Klassifizierung immer ätiologische Momente. Die verschiedenen Theorien über die Ursachen des Wahns fließen daher auch bei Lacan in die diagnostischen Kriterien ein. Er entwickelt seine Kriterien in mehreren Phasen: Zunächst in den ersten Jahren seiner beginnenden öffentlichen Wirksamkeit in den Zwanziger- und Dreißigerjahren des vorigen Jahrhunderts, dann in seinen früheren Seminaren in den Fünfzigerjahren und schließlich in seinen späten Seminaren in den Siebziger Jahren. Corinne Fellahian unterscheidet vier formale Zugangsweisen Lacans, in deren Rahmen er unterschiedliche Modelle verwendet: Die Persönlichkeit (1932), die Komplexe (1936), die Verwerfung (1955-1958) und den borromäischen Knoten (1974-1976) (Fellahian 2005). Massimo Recalcati sieht in Lacans Beschäftigung mit der Ätiologie des Wahnsinns, mit welchem Begriff Lacan die Psychose in Anlehnung an eine in den Humanwissenschaften übliche Vermischung von Wahn und Psychose bisweilen bezeichnet, vier verschiedene Themenfelder: Freiheit, Ausschluss des Anderen, ödipales Scheitern, universelle Verbreitung des Wahns (Recalcati 2008). Die mit diesen Eckpunkten äußerst weit gespannte Thematik enthält die schon erwähnte Doppelseitigkeit von Lacans Sicht auf den Wahn. Wahn und Wahnsinn bedeuten für ihn gleichermaßen das Anstoßen an eine Grenze (was durch den Ausschluss des Anderen oder ein ödipales Scheitern beschrieben werden kann), wie die Eröffnung von Möglichkeiten, die sich in einer Vorstellung vom Wahnsinn als Ausdruck menschlicher Freiheit oder vom Wahn als sprachlich normales, ja teilweise universales Phänomen darstellen.

Wir werden hier die verschiedenen Phasen lacanscher Theorieentwicklung zur Psychose und zum Wahn mit vier anderen Begriffen in Verbindung bringen, in denen sich dieser Perspektivenwechsel manifestiert: lebensgefährlicher Kampf, verbietender Vater, unsicheres Geschlecht und produktive Schrift. Diese vier Facetten des Wahns, die in ätiologische Modelle eingearbeitet auftreten, berücksichtigt Lacan in den verschiedenen Phasen seiner Auseinandersetzung mit der Psychose unterschiedlich stark. Während in seinen früheren Arbeiten Fragen bezüglich des Kampfes dominieren, in seinen mittleren Arbeiten das Vaterthema und die Geschlechtsunsicherheit wichtiger werden, bekommen das Schreiben und die Schrift in der letzten Phase seiner theoretischen Entwicklung besonderes Gewicht in seiner Auseinandersetzung mit Wahnhaftem und Psychotischem. Das bedeutet freilich nicht, dass die 
anderen Themen in den jeweiligen anderen Phasen verschwinden: Schon sehr früh hat sich Lacan mit der Schrift in psychotischen Zuständen befasst (Lévy-Valensi et al. 1931) und auch in seinen späten Arbeiten sind das Geschlecht und der Vater wesentliche Bezugspunkte für seine Überlegungen.

\subsection{Lebensgefährlicher Kampf}

Lacan hat viele Jahre im Bereich der forensischen Psychiatrie gearbeitet und hat sich in mehreren Texten mit forensischen Fallgeschichten befasst (z.B. Lacan 1933a, 1975a). Seine dabei entstehenden Auffassungen zu Psychose und Wahn sind von den Eckpunkten einer psychiatrischen Ausbildung bestimmt - etwa von der Frage, ob eine organogenetische Disposition zur Psychose gehört oder ob die Psychose als ein Problem der Persönlichkeit anzusehen ist (Lacan 1975a). Zu den Themen der psychiatrischen Ausbildung gesellte sich sein Interesse an philosophischen Fragen, im Rahmen dessen ihn in den 193oer-Jahren Alexandre Kojèves Lektüre von Hegels Herr-undKnecht-Dialektik beschäftigte. Hier finden sich die Motive der Freiheit und der kämpferischen Aggressivität, die für seine frühen Konzepte zum Wahn und zur Psychose wichtig werden.

In seiner Dissertation entwickelt er 1932 anhand einer Messer-Attacke, die eine seiner psychiatrischen Patientinnen auf eine Schauspielerin verübt hatte, das Konzept der Selbstbestrafungsparanoia. Der heuristische Wert dieses Konzepts liegt vor allem darin, einem Wahn bei einer fakultativ vorhandenen somatischen Verursachung einen sinnhaften Zusammenhang mit der Persönlichkeit des Erkrankten zuzuschreiben (Fellahian 2005). Ätiologisch sind für den Ausbruch einer Selbstbestrafungsparanoia mehrere Momente zu unterscheiden: Gelegentlich vorkommende organische Ursachen, ein moralischer Konflikt meist gegenüber Eltern oder Geschwistern, häufig assoziiert mit einer traumatisch erfahrenen Lebensveränderung, sowie eine spezifische Fixierung in der Entwicklung des Überichs oder des sekundären Narzissmus. Die Gefährlichkeit, die von einem solchen Subjekt ausgeht, verdankt sich einer spezifischen Konstellation: Wahnhafte Selbstbeschuldigungen und eine Tendenz, diese Selbstbeschuldigungen in einem passage-à-l'acte agierend gegenüber anderen in Attacken zu realisieren (Lacan 1975a).

Patient 1 (Herr S.) bedrohte seine Mutter und/oder attackierte Bewohner des Pflegeheimes, in welchem seine Mutter wohnte - der Fallbericht bringt beide Versionen als außenanamnestische Darstellung (Stompe 2010). Herr S. selbst spricht von einem planvollen Vorgehen, das darauf ausgerichtet gewesen sei, die Polizei zu einer Handlung gegen ihn zu nötigen, in deren Rahmen er stirbt. Inwieweit die Schmerzen, von denen er dabei erlöst werden wollte, einer Rationalisierung von Schuldgefühlen entsprechen, lässt sich ohne den Patienten nicht beurteilen. Jedenfalls ist der Schritt zur Tat, der passage-à-l'acte, den er gegen sich selbst gerichtet sehen wollte, zunächst projektiv auf andere bezogen. Außerdem endet mit diesem Schritt die floride Phase seiner Psychose.

Darin ist sie einem anderen Fall ähnlich, der die französische Öffentlichkeit 1933 beschäftigte: Das Verbrechen der Schwestern Papin (Lacan 1933a). Die beiden Hausmädchen haben im Rahmen einer psychotischen Dekompensation ihre Dienstgeberin und deren Tochter auf grausame Weise ermordet. Lacan deutet den Wahn, der diesem Geschehen zugrunde liegt, als Folge einer aus einer unterdrückten Geschwister- 
rivalität resultierenden narzisstischen Fixierung der beiden Schwestern aufeinander. Die gegenseitige homosexuelle Bindung der Schwestern konnte aufgrund ihrer Intensität nur gewaltsam - durch Tötung der projektiv als Doppelgängerinnen aufgefassten Dienstgeberin und ihrer Tochter - gelöst werden. Wie bei Herrn S. endet auch bei den Schwestern Papin die offene Manifestation der Psychose mit der Tat. Eine Besonderheit an Lacans Diskussion dieses Falles ist die Einbeziehung der sozialen Realität der beiden wahnsinnigen Schwestern (Roudinesco 1993). Sie werden zu Protagonistinnen eines Klassenkampfes, der Züge jenes Kampfes zwischen Herrn und Knecht trägt, den Hegel für die Herausbildung des Bewusstseins beschreibt.

In allen drei Fällen - bei der Messerattacke der Patientin, dem Mord der Schwestern Papin und bei Herrn S. - wird eine (para-)suizidale Handlung an anderen vollzogen. Wie ist das zu verstehen? Die Verwechslung zwischen dem Ich und dem anderen ist Teil einer frühen Entwicklungsphase des Subjekts. Lacan hat diesen Aspekt in seine Theorie des Spiegelstadiums als Verwechslung mit dem Spiegelbild aufgenommen (Lacan 1973). Was als narzisstische, homosexuelle Fixierung imponiert, ist die Oberfläche einer äußerst spannungsgeladenen, aggressiven Gesamtsituation, in welcher das Ich, um sich vom anderen zu lösen, bereit ist, mit dem anderen einen Kampf auf Leben und Tod zu führen. Eine Verhandlung mit dem anderen ist an dieser Stelle nicht möglich. Dazu reicht die Sprachentwicklung nicht. Die Verwechslung richtet sich nicht zufällig auf das Bild, die imaginäre Repräsentation des anderen. Es gilt hier nur ein „Entweder-Oder“, „entweder ich oder der andere“. Beim Wahn imponiert ein solches imaginäres Moment von Rivalität als Unkorrigierbarkeit. Auch der Kampf um eine wahnhaft strukturierte sprachliche Bedeutung ist einer, der von Antagonismen bestimmt ist: Entweder die eine oder die andere Deutung ist korrekt. Wer sich korrigieren lässt, unterliegt.

Die Tötung des anderen, die einen Suizid vertritt, offenbart einen jedem Subjekt eingeschriebenen Kampf. Herr S. verdeutlicht ihn noch mittels eines anderen imaginären Elements seines Wahns, das an die Schwestern Papin erinnert: Es ist sein Zwillingsbruder, der als Schatten, als geheimer Doppelgänger (Rank 2007) des Subjekts auftaucht. In Herrn S.' Wahn lebt der Zwillingsbruder ein paralleles Leben als verwechseltes Kind einer anderen Familie, wird von Herrn S. befreit und wenig später von dessen Frau getötet. Es sieht so aus, als zeichne sich auch an diesem Bruder die verfehlte Subjektentwicklung des Patienten ab: die Identifikation mit einem Ähnlichen, mit dem sich Herr S. verwechselt, die Rivalität, die sich zunächst als moralisch wünschenswerte Handlung tarnt und schließlich durch den Mord zu erkennen gibt. Die zur Subjektbildung erforderlichen Schritte der Entfremdung vom Gleichen (l'alienation) und der Trennung vom Ähnlichen (la separation) können nicht erfolgen.

\subsection{Verbietender Vater}

Im Anschluss an Überlegungen, die er 1938 in einem Lexikonartikel darstellt (Lacan 1980a) entwickelt Lacan in seinem Seminar Die Psychosen den Gedanken, dass der zentrale Mechanismus für die Entstehung einer psychotischen Struktur in einer speziellen Störung der ödipalen Entwicklung zu sehen ist (Lacan 1997). Die duale Beziehung des Kindes zur Mutter nimmt deletären Charakter für ein Kind an, wenn es nicht gelingt, aus der dualen Beziehungskonstellation zwischen Mutter und Kind eine trianguläre zu formen. Dazu muss das Kind erfahren und anerkennen, dass die müt- 
terliche Wunschökonomie, das mütterliche Begehren, nicht auf es allein, sondern darüber hinaus auf den Vater als den Anderen gerichtet ist. Anders gesagt: Das Kind, das bis zu dieser Erkenntnis seine Mutter begehren konnte, muss eine Relation zum Vater entwickeln. Dazu ist es nötig, dass die Mutter ein (begehrendes) Verhältnis zu einem als Vater fungierenden Objekt hat und dass das Kind in diesem mütterlichen Objekt ein Ichideal annehmen kann, um ein Überich zu entwickeln, was Lacan zwei Jahre nach dem Seminar über die Psychosen detailliert darstellt (Lacan 2006). Schließlich präsentiert sich diese Relation im Hinblick auf die Mutter als ein Verbot im Sinne einer Untersagung: Der Inzest mit der Mutter, das unstrukturierte Genießen, gilt als untersagt von der väterlichen Instanz.

Die Struktur eines Subjekts ist nicht nur im Imaginären, sondern auch im Symbolischen aus den Koordinaten des Anderen gebildet: Der Andere und der Diskurs, der von diesem Anderen bestimmt wird, ist als Rahmen dafür anzusehen, ob ein Subjekt psychotisch oder neurotisch geworden sein wird (Lacan 1975). Da Lacan die Vaterfigur als den Hauptrepräsentanten der Sprache und damit der symbolischen Ordnung versteht, lässt sich die Anbahnung des Verhältnisses zum Vater als eine Einführung des Kindes in die Sprache begreifen. Dabei ist das wirksame Agens zur Trennung von Mutter und Kind nicht der leibhaftige Vater, sondern das, was den Vater in der symbolischen Ordnung repräsentiert: sein Name, der für ein Gesetz, nämlich das Inzesttabu, steht, und der metaphorisch auch andere Signifikate bezeichnen kann, die im Ernstfall diese trennende Funktion erfüllen können wie beispielsweise den Beruf der Mutter, deren Lieblingsbeschäftigung oder andere Subjekte, die dem Kind den Platz in der Beziehung mit der Mutter streitig machen.

Die Trennung von der Mutter ist ein lang andauernder Prozess, in dem es wesentlich ist, dass der Name des Vaters im Imaginären des Kindes als der Ort einer Orientierung für die Bedeutungsgenese aufgerufen und verankert wird. Diese Aufrufung (l'évocation) kann auf verschiedene Weisen scheitern (Ver Ecke 2009): Bei Hölderlin war es seine Mutter, die in ihrer Trauer um den toten Gatten mit ihrem Sohn nicht zu sprechen in der Lage war, an diesen daher auch den Namen des Vaters nicht in sprachlicher Form herantragen konnte. Pierra Aulagnier hat psychotische Strukturen in Zusammenhang mit der Unfähigkeit von Müttern gesehen, sich den sexuellen Umstand der Herkunft ihres Kindes einzugestehen, was einen Aufruf der väterlichen Metapher im Keim verhindert (Aulagnier 1975). Fink macht psychologische Eigenschaften des Vaters namhaft, die einem werdenden Subjekt von der imaginären Achse aus die Einbeziehung der symbolischen schwer machen: Ein vor allem rivalisierender Vater, der eine herrschsüchtige, monströse Position einnimmt, weist dem Kind eine aussichtslose Rolle zu (Fink 1999). Danielle Bergeron hat einen anderen Ansatz: Sie sieht die fehlende Bedeutung, die individuellen Vätern im Laufe von Generationen zukommt, als trigger für eine psychotische Entwicklung (Bergeron 1990). Damit kommt sie Lacans Überlegung aus dem Seminar über die Psychosen sehr nahe: Die Aufrufung des väterlichen Namens kann auch durch den Vater selbst verhindert werden, indem dieser beispielsweise als gottähnlicher Vertreter eines mächtigen Gesetzes persönlich versagt (Lacan 1997).

Was ist der Zweck der Installierung des väterlichen Namens im symbolischen Universum eines kindlichen Subjekts? Zum einen wird dem Vater in diesem Prozess Macht zugeschrieben, die als phallische Macht den Wert eines Organisators des kindlichen Begehrens erhält (Ver Ecke 2009). Das Kind identifiziert sich mit dem Vater, 
den es deswegen als Ichideal auffasst, weil er in jener Position der Mutter gegenüber ist, die das Kind gerne einnehmen will (Lacan 2006). Der Vatermetapher kommt die Aufgabe zu, die kindliche Libido neu zu strukturieren. Das Genießen des mütterlichen Körpers - ein Genießen, bei dem das Kind gleichermaßen Gegenstand wie Akteur ist - wird durch phallisches Genießen ersetzt. Die Verwerfung des Vaternamens, die Lacan 1956 ins Zentrum der psychotischen Entwicklung rückt, wird zunächst als eine Alles-Oder-Nichts-Bewegung beschrieben. Im Lichte der späteren theoretischen Entwicklung Lacans ließe sich diese Bewegung auch graduell - mehr oder weniger vollzogen - denken: Das Genießen der Mutter würde im psychotischen Fall nur teilweise in ein phallisches Genießen überführt (Ver Ecke 2009). Wenn die Installation des Namens des Vaters nicht gelingt, kommt es jedenfalls zu einer Deformation der strukturbildenden Register im Subjekt, was Lacan graphisch darstellt (Lacan 1975):

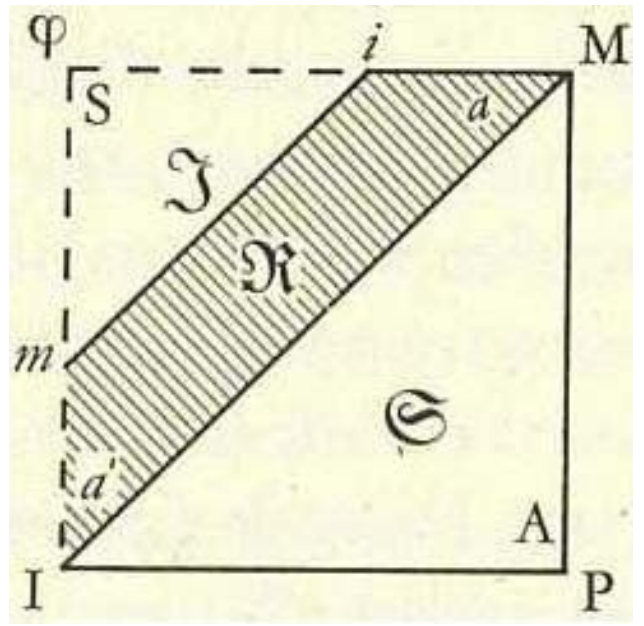

Abb. 10 Schema R

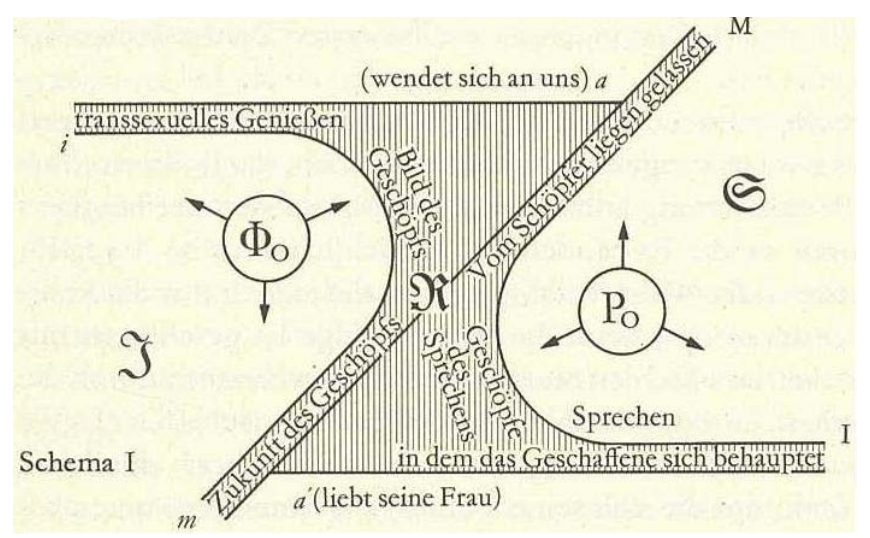

Abb. 11 Schema I 
Im Schema R (s. Abb. 10) wird die reale Basis des nicht-psychotischen Subjekts, das Spiegel-Ich in seiner dialektischen Verfangenheit mit dem mütterlichen Pol samt ihrer imaginären und stets wahnhaften Umformung links oberhalb der Diagonale zwischen I und M abgebildet. Das Subjekt, das sich gleichsam als Echo, als Evokation der Vatermetapher im Imaginären ausbildet (Lacan 1975), findet sich als Phallus links oben vis à vis der väterlichen Metapher $\mathrm{P}$, die am symbolischen Ort des Anderen rechts unten installiert ist. Schrebers Psychoseerfahrungen bilden den Inhalt des Schema I (s. Abb. 11): Der Pol des Anderen fehlt hier ebenso wie der Pol des Subjekts. Das Genießen bedarf - im Unterschied übrigens zum Begehren (Thibierge 2003) - keines Subjekts. Das mütterliche Genießen, die reale Matrix psychotischer Erfahrung, die der gesamten schraffierten Fläche entspricht, wird von zwei Seiten geformt, nicht aber begrenzt: einerseits von den Effekten einer phallischen Wahnmetapher in einem zusammengebrochenen Imaginären (Lacan 1975) und andererseits von einem durch einen wahnhaften Vaternamenersatz bestimmten Sprechen im Bereich eines nicht etablierbaren Symbolischen geformt, nicht aber begrenzt.

Damit ist ein weiterer Zweck der Installierung des Vaternamens am Ort des Anderen genannt: Ein sogenannt normales, nicht psychotisches Subjekt kann sich der spezifischen symbolischen Funktion der menschlichen, d.h. artikulierten Sprache bedienen. Diese ist durch eine Gleitfähigkeit zwischen Signifikant und Signifikat gekennzeichnet, die durch eine zentrale Metapher (Vatername) möglich gemacht ist und die ein Subjekt bestimmt, das sich metaphorisch als Phallus zu fassen und imaginär zu rahmen versucht - ein, nebenbei gesagt, in seiner Phallozentrizität nicht unwidersprochenes Konzept von Sprache (Lacoue-Labarthe u. Nancy 1973). Das hat Folgen für das Sprechen: Psychotische Subjekte zeigen oftmals einen Hang zum Konkretismus und können sich der metaphorischen Funktion der Sprache nicht bedienen (Fink 1999). Nicht-psychotische Subjekte dagegen können sich ohne Schwierigkeit einen Träger des Namens Jean-Paul Sartre vorstellen, der kein französischer Philosoph, sondern etwa ein Hausbesorger im Paris der Neunziger Jahre ist. Der Name „Sartre“ gleitet dabei von einem Signifikat zum anderen.

Dass solche Übertragungsphänomene nicht zu einem Zusammenbruch der Sprechmöglichkeiten führen, hat damit zu tun, dass sich eine Verankerung sämtlicher bedeutungsgenerierender Prozesse am Pol der Vatermetapher findet. Lacan denkt diesen Signifikanten ohne eine fixe Bedeutung, nennt ihn daher „leer“. Dieser Signifikant muss ohne Bedeutung sein, damit alle übrigen Signifikanten Bedeutung erlangen. Das Sprechen des neurotischen Subjekts ist von einer Art Sog gekennzeichnet, der von dieser Leerstelle ausgeht. Indem diese sich als stets neu besetzbare, aber letztlich unbestimmbare erhält, stabilisiert sie ihren eigenen Sog, der sich im Gleiten der Signifikanten darstellt. Ist die väterliche Metapher nicht als grundlegende angenommen - Lacan spricht in diesem Zusammenhang von einer Verwerfung der väterlichen Metapher - so fehlt der Sprache insgesamt ihre übliche metaphorische Funktion. Anstelle der väterlichen Metapher bildet sich ein Loch (Lacan 1975). Signifikate können nicht in der üblichen Weise gleiten und damit auch nicht anderen Signifikanten unterlegt werden. In der Signifikantenkette steht mit diesem Loch etwas auf dem Spiel, was durch das Auftauchen von Wahnbildungen zum Ausdruck kommt und gleichzeitig durch solche fixen Wahnüberzeugungen auch in Schach gehalten wird (Apollon 2002). Im Bemühen, Ersatz für die väterliche Metapher, den phallischen Signifikanten, und damit ein Zentrum der eigenen Bedeutungszuschreibung zu finden, treten wahnhafte Fixierungen auf. Die Vorstellung Schrebers, Gott im Beischlaf 
zu unterliegen, ist Lacans Beispiel für eine aus der fehlenden Insertion der Vatermetapher resultierende Wahnbildung.

Geneviève Morel hat Lacans Ansatz, insofern er sich an der väterlich bestimmten Sprache orientiert, aufgenommen und weitergeführt (Morel 2007, 2008). Sie bezieht sich auf eine Bemerkung Lacans in seinem Seminar V, in welcher er neben dem Gesetz des Vaters von einem Gesetz der Mutter spricht (Lacan 2006). Damit meint er, dass jene sprachlichen Ausdrücke, die früh an ein Kind gerichtet werden, unter Umständen sehr bestimmend, ja prägend werden können und in diesem Sinne gesetzesförmigen Charakter bekommen. Ihre prägende Kraft verdanken sie der sprachlichen Entwicklungsstufe des Kindes: Sie werden konkret genommen, da die Fähigkeit, Metaphern zu verstehen, in den ersten Lebensjahren fehlt. Morel hat bei Patient/-innen mit psychotischer Struktur regelmäßig solche wahnförmig funktionierenden Sätze von Mutterfiguren gefunden, deren oftmals aggressiver Inhalt im Rahmen von psychotischen Exazerbationen agiert wird. Morel erzählt etwa von einer Krankenschwester (Morel 2007), die ihre Kinder und mehrere Patient/-innen zu töten versucht, da sie ihre Existenz mit dem, von ihre konkretistisch aufgefassten mütterlichen Satz „Du hättest nicht leben sollen“ verbindet. Ihre Mutter hatte diesen Satz nach der sehr gefährlichen Geburt der Tochter immer wieder geäußert.

Herrn S.' Angriff auf seine Mutter (vgl. Stompe 2010) würde Anlass bieten, hellhörig auf solcherlei wahnhafte Sätze in seinem Sprechen zu achten. Seine Vorstellung, sein eigentlicher Vater wäre Adolf Hitler gewesen, lässt sich als ein Prozess begreifen, der dem Muster einer Wahnbildung nach Verwerfung der väterlichen Metapher folgt: Eine Insertion der väterlichen Metapher ist aus Gründen, auf die das vorliegende Material keinen Rückschluss zulässt, nicht erfolgt. Die physische Abwesenheit des Vaters nach der Trennung der Eltern kann diesen Prozess nicht erklären, weil, wie bereits gesagt, die Verankerung der väterlichen Metapher keines konkreten Vaters bedarf. Herr S. versucht wie Schreber die fehlende Leerstelle durch verschiedene Vorstellungen von einem ganz besonderen Vaterobjekt zur Geltung zu bringen. Dieser Vater erweist sich als gefährlich und bedrohlich. Deutlich trägt er gleichzeitig Züge des ersten Objekts, einer ebenfalls als gefährlich erlebten Mutterfigur. Als Phallusersatz steht Herr S. dieser Mutter gegenüber.

In narzisstischer Rivalität verbannt Herr S. in seinem Wahn das Vaterobjekt aus seinem eigenen Umkreis, was ihm angesichts der Deformation seines Sprechens möglich ist. Weder der Bezug zu einem Signifikat noch zu einem externen Referenten kümmern Herrn S. Anders als andere Sprecher kann und braucht sich Herr S. nicht auf einen Prozess einzulassen, in dem die Bedeutung eines Zeichens wie „Hitler“ im Sprechen mit anderen Sprecher/-innen festgelegt oder verschoben wird. Anstelle von gemeinsamen, mit einer Sprachgemeinschaft geteilten Signifikanten treten die wahnhaften, „wahren Namen Gottes“. So lässt sich auch die Gestalt des nächsten Vaternamens begreifen, dessen Herr S. sich bedient: „Jah, preisen tut weh“ ist eine am verbalen Ausdruck orientierte private Umgestaltung des hebräischen Gottesnamens Jahwe, der als ein Vaternamenersatz fungiert. Dessen Ersatzfunktion wird in der Wahnerzählung konkret deutlich gemacht: Er ist ersetzbar durch „Komm, rüste uns“. Die Form dieses Ersatzes unterscheidet sich vom Signifikantengleiten des Neurotikers, denn Herr S. bestimmt sie: Er allein setzt die neue Bedeutung fest - ohne jeden Bezug zu anderen Sprecher/-innen. 
Herr S. ist nicht in der Lage, ein väterliches von einem mütterlichen Objekt zu unterscheiden. Die Größe der väterlichen Figuren kann als Ausdruck eines Wunsches von Herrn S. verstanden werden: Es möge jemanden geben, der ein übergroßes und mit 120 Enkeln von ihm selbst als fruchtbar imaginiertes Genießen begrenzt. Die sich von Schrebers Wahn unterscheidende Rivalität mit dem Objekt bestimmt die Form des Genießens, die Herr S. anspricht: Als jemand, der viele Frauenbekanntschaften und die meisten davon geheiratet hat, scheint er es mit Freuds Urvater aufnehmen zu wollen, dem alle Frauen zur Verfügung stehen. Auch seine Vorstellung, bestimmt zu sein, die wahre Bibel nach Österreich zurück zu bringen, enthält ein Moment von narzisstischer Selbstüberhöhung im Kampf mit einem als übermächtig imaginierten Objekt. Seine Auserwähltheit, eine antifaschistische Bewegung zu gründen, die ihn schließlich am Suizid hindert, macht deutlich, dass es auch zwischen dem Vaterobjekt und ihm um Leben und Tod geht.

\subsection{Unsicheres Geschlecht}

Da die Identifizierung mit einem Geschlecht eine Folge von Identifizierungs- und Desidentifizierungsschritten mit den elterlichen Objekten voraussetzt, wirkt sich die Störung auf der Achse zum Vater auch auf die Vorstellung vom eigenen Geschlecht aus. Eine Ambiguität dem eigenen Geschlecht gegenüber, wie sie in vielen psychotischen Entwicklungen vorkommt, überrascht daher nicht. Sowohl hinsichtlich einer bestehenden Ambiguität als auch hinsichtlich einer Identifizierung mit dem je anderen Geschlecht bestehen Unterschiede zwischen der weiblichen und der männlichen Position.

Im Ausgang von Freuds Arbeit über die Autobiographie des Gerichtspräsidenten Schreber (Freud 1911) arbeitet Lacan in seinem Seminar über die Psychosen die Frage nach dem eigenen Geschlecht als einen Knotenpunkt für die psychotische Struktur aus. Schreber hält sich in seiner Psychose wahnhaft für eine Frau - die Frau Gottes, die diesem im Beischlaf unterliegt. Im Unterschied zu Freud, der Schrebers Verweiblichung in Zusammenhang mit einer mangelnder Abwehr homosexueller Strebungen begreift, sieht Lacan die Feminisierung in den 5oer-Jahren als ein strukturelles Moment (Fink 1999), das sich auf den Phallus bezieht. Schreber findet sich in der Position Phi-Null als Phallusersatz (s. Abb. 11). Er fungiert im Imaginären wahnhaft als eine Frau, die das begehrte Objekt jener Struktur ist, die sich als P-Null auf der Seite des Vaternamens im Symbolischen findet. Schreber unterliegt nicht deswegen einer Verweiblichung, weil er sich im Gefolge seiner Verwerfung des Vaternamens vom Penis distanziert hat (Lacan 1975), vielmehr gerät er aufgrund der Identifizierung mit dem Phallus im Wahn auf eine weibliche Seite, gelten Weiblichkeit und Männlichkeit Lacan doch als zwei Positionen, die sich voneinander durch Sein oder Haben des Phallus unterscheiden (Lacan 1975b).

Die partielle Falldarstellung von Herrn S. verrät nicht viel über seine geschlechtlichen Identifizierungen. Seine Vorstellung, dass die Eigenschaften gut, logisch und männlich so zusammengehören wie böse, unlogisch und weiblich, deutet an, dass er die Geschlechter nicht als gleitende Signifikanten denken, sondern sie wahnhaft fixierend als Stepppunkte behandeln muss. Allerdings ist ein solcher Umgang mit Geschlechterstereotypen auch unter Menschen mit einer nicht-psychotischen Struktur anzutreffen, sodass der pathognomonische Wert dieses Wahninhaltes dahingestellt 
bleiben muss. Für Herrn S. steht die Frage seines Geschlechts jedenfalls nicht im Vordergrund seiner Wahnbildungen. Anders bei Frau S. (Patientin 2). Sie hält sich für Jean Paul Sartre und verbindet dies mit der Überzeugung, über einen Penis zu verfügen. Wie lässt sich diese Wahnbildung einer Vermännlichung mit Lacan verstehen?

Über Frau S.' Vorgeschichte wird in der Vignette nicht viel gesagt. Der folgende Gedankengang ist daher mehr als eine theoretische Konstruktion anzusehen, die eine männliche Identifizierung im Rahmen einer psychotischen Struktur verständlich machen kann. Mann und Frau bzw. eine strukturell männliche und eine strukturell weibliche Position werden psychoanalytisch nicht als symmetrisch und auch nicht als einander ergänzend aufgefasst. Nicht ohne Einspruch vonseiten der weiblichen Analytikerinnen steht für Freud und auch für Lacan allein das männliche Organ, der Penis bzw. der Phallus, im Zentrum des psychosexuellen Reifungsprozesses, was sich im Kastrationskomplex paradigmatisch darstellt (Lacan 1975b). Mit seiner Formel der Sexuierung zeigt Lacan, dass sich die Verhältnisse auf der Seite des Mannes von jenen aufseiten der Frau unterscheiden: Die Subjekte, die sich auf der männlichen Seite einordnen, können sich auf eine Ausnahme von der phallischen Funktion stützen, nämlich den Urvater, der nicht auf den Phallus und die mit diesem verbundenen Einschränkungen angewiesen ist. Auf der Seite der Frauen existiert eine solche Ausnahme nicht. Kulturell wird keine Urmutter imaginiert. Keine Frau hat - solange sich alles um den Phallus dreht - eine eigene Position. Das ist der Grund, dass die weibliche Seite auf die Seite des Mannes anders angewiesen ist als umgekehrt. Die weibliche Seite, das Weibliche allein existiert nicht (Lacan 1986) in einem Universum, das männlich gedacht wird (Kadi 2002, 2006).

Der von Lacan mit Phi bezeichnete symbolische Phallus ist als Zeichen der Differenz insofern privilegiert, als er die Differenz der Geschlechter als eine für jeden Menschen grundlegende Differenz markiert. So betrachtet werden sowohl Mann als auch Frau durch ein Geschlechtsmerkmal gekennzeichnet. In diesem Sinn haben beide Anteil am Phallus. Da dieser aber durch seine imaginäre Identifizierung mit dem Penis stärker am männlichen Geschlecht haftet, gerät die Frau in eine größere Distanz zum Symbolischen und hat daher als Die Frau im Sinne einer Gesamtheit keine Existenz im Symbolischen (vgl. Castanet 1994; Ruhs 1995). Gleichzeitig hat die einzelne Frau aber Zugang zu einem anderen, gegenüber dem phallischen als überschießend zu bezeichnenden Genießen, auf das sie sich außerhalb der phallischen Ordnung beziehen kann.

Die Geschlechterordnung bringt Lacan 1973 in Verbindung mit dem Genießen in der Psychose. Indem er neuerlich auf Schrebers Verweiblichung zurückkommt, wird das andersartige, sogenannte weibliche Genießen mit dem psychotischen Genießen, welches er nun als pousse-à-la-femme (Verweiblichungsschub) bezeichnet (Lacan 1973a), enggeführt. Für Fink gibt Lacan den Unterschied zwischen psychotischem und weiblichem Genießen nie ganz auf (Fink 1999), während Morel ein Zusammenfallen von beiden Formen des Genießens postuliert (Morel 2000). Sie sieht die Verwerfung des Namens-des-Vaters in der Psychose in Eins fallen mit der Existenzmöglichkeit der Frau. Und Schrebers Wahn gerät Morel zufolge in eine Kontinuität zu einer nicht exazerbierten Psychose mit einer ausgeprägten Identifizierung mit der Mutter. Anders gesagt: In der Psychose existiert das anders nicht existente Weibliche über sein Genießen. Dieses Genießen ist ein fürchterliches Genießen.

So wird verständlich, weshalb sich bei Frau S. eine Bewegung einer Identifizierung mit Jean-Paul Sartre als einer männlichen Position einstellt. Sie lässt sich als Flucht 
vor einem weiblichen Genießen in seiner schrecklichen, weil unbegrenzten Dimension begreifen. Frau S. sucht im Imaginären eine solche männliche Position, eine Position des „Phallus-Habens“. Dies impliziert eine partielle Identifizierung mit dem Vater, worin neuerlich erkennbar wird, dass die psychotische Struktur nicht so sehr auf einem in der Realität mangelnden Vater, sondern auf einer mangelnden Vatermetapher, einer mangelnden Funktion des Vaternamens im Symbolischen, beruht. Frau S.' Vorstellung, über einen Penis zu verfügen, rückt den protektiven Aspekt des Wahns in den Vordergrund, kann ihr Versuch, dem fürchterlichen Genießen durch eine Vermännlichung zu entkommen, doch als Selbstheilungsversuch angesehen werden, mit welchem sie einer Verwerfung der Vatermetapher entgegenarbeitet.

\subsection{Produktive Schrift}

Eine deutliche Veränderung erfährt Lacans Theorie des Wahns durch eine theoretische Wende, die in seinen Arbeiten erstmals 1960 auftaucht (Lacan 1991). Lacan erwähnt hier, dass der große Andere, für den der Name des Vaters steht, über keine weitere symbolische Stütze verfügt. Bereits in den 1930er-Jahren hatte Lacan die Vaterfunktion als eine beschrieben, die in unserer Kultur unsicher geworden ist, was sich etwa in einer zunehmenden Säkularisierung (die auch von einer oftmals ins Wahnhafte übersteigerten Religiosität nicht infrage gestellt werden kann) darstellt. Und da sich für ein einzelnes Subjekt am Horizont seiner Subjektivität stets die Auffassung von Subjektivität, die in seiner Zeit als gegeben gilt, auftut (Lacan 1975), ergeben sich aus diesen veränderten kulturellen Bedingungen auch andere Subjektivierungsbedingungen. Die kulturellen Veränderungen bilden allerdings nur den Hintergrund der Subjektstruktur. Ob ein großer Anderer kulturell supponiert wird oder nicht, kann nicht Thema einer klinischen Behandlung sein (Kadi 2011), und die Dramatik des Verfalls der Vaterfunktion wird von manchen auch in Zweifel gezogen (Fink 1999).

Lacans Annahmen über die Signifikantenordnung erfahren jedenfalls mit dieser Wende, die eine Abwendung vom Paradigma eines großen Anderen darstellt, eine weitreichende Veränderung. Galt ihm in den Sechziger Jahren das leere Zentrum des phallischen Signifikanten noch als eines, das dieser Ordnung durch die Dynamik einer angestrebten Füllung der leeren Mitte eine Stabilität verliehen hat, so vertritt dieser Signifikant nun das Scheitern des Sinns (Lacan 1986). Lacan versucht den veränderten Bedingungen mit einem neuen Konzept zu entsprechen, in dem der Andere keine konstitutive Rolle für ein Subjekt mehr einnimmt (Skriabine 2009): das Konzept des Borromäischen Knotens. Er denkt das Subjekt nun als eine mehr oder minder geglückte Verknüpfung der Register des Symbolischen, des Imaginären und des Realen, in der der Andere nur ephemer auftaucht. Die Aufgabe, die das Subjekt für seine Subjektivierung zu lösen hat, ist nicht verändert: Es gilt, dem grenzenlosen Genießen zu entkommen, wozu beispielsweise ein Genießen des Anderen, des Sinns oder des Phallus bereit stehen (Lacan 2005).

Doch anders als in den früheren Ansätzen ist der Name des Vaters, der die Benennungsfunktion in der Sprache vertreten hat, nun keine privilegierte Lösung mehr, um der Psychose zu entkommen. Der Name des Vaters hat sich vervielfältigt und ist nicht mehr nur über das Symbolische wirksam (Skriabine 2009). Auch von den anderen Registern aus wird versucht, die Aufgabe der Benennung zu übernehmen. Die Hemmung entspricht dabei einer Benennung im Imaginären, das Symptom einer 
Benennung im Symbolischen und die Angst einer Benennung im Realen (Lacan o.J.). Wesentlich an dieser Verschiebung des gesamten theoretischen Rahmens ist das Moment der Vervielfältigung: Es gibt nicht mehr eine einzige Form der Stabilisierung eines Subjekts, sondern eine Vielzahl an möglichen Verknüpfungen. Der Ödipuskomplex ist einer unter mehreren vierten Ringen, die die borromäische Dreierkonstellation zu stabilisieren in der Lage sind (Lacan o.J.).

Lacan beschreibt seinen Weg als einen, der vom „sym, qui bole“, zum „sym, qui ptôme" führt (Lacan 2005a). Nicht das Symbol, das Wort, der Signifikant, sondern das Symptom, das wie ein Wahngebilde strukturiert sein kann, gilt ihm in den 197oerJahren als das, was einem Subjekt die notwendige Stütze vermitteln kann. Anhand des Werks von James Joyce stellt Lacan Entwicklung, Form und Zweck des Symptoms, das er Sinthom nennt, dar. Reales, Symbolisches und Imaginäres werden dabei durch drei mehr oder minder zusammenhängende Ringe topologisch verkörpert. Anders als im Psychosenseminar, in welchem Lacan von einem Konzept einer normalen Entwicklung (s. Abb. 10) ausging, um in Abhebung davon eine pathologische Entwicklung darzustellen (s. Abb. 11), denkt er nun den Ausgangspunkt als pathologische Konstellation, die mithilfe des Symptoms stabilisiert werden soll. Das Symptom kann Fehler in der Verbindung der drei Register korrigieren, indem es wie ein vierter Ring die anderen subjektiven Modalitäten zusammenhält (s. Abb. 12 a, b) (Lacan 2005):

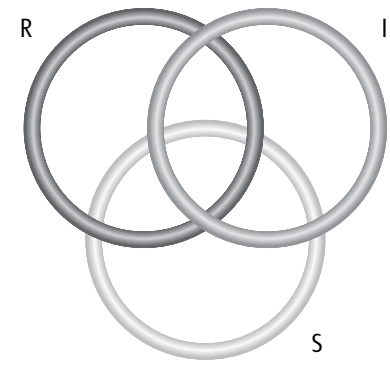

(a)

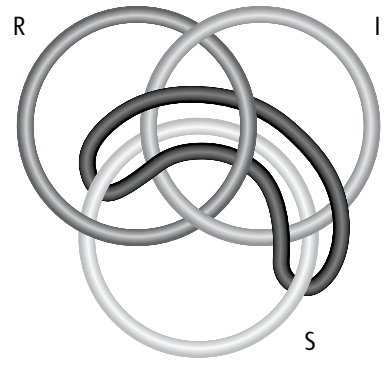

(b)

Abb. 12 a, b Drei unverbundene Ringe und Sinthom als vierter Ring (R: Reales, S: Symbolisches, I: Imaginäres)

Nicht selten ist einer Psychose ein Reichtum an literarischer Produktion eigen (Fink 1999). Joyce' Symptom ist sein Schreiben. Dieses Schreiben unterscheidet sich deutlich von anderen Formen des Schreibens, denn es bedient sich einer kreativen Umformung bekannter sprachlicher Ausdrücke, was zu einer erheblichen Erschwerung des Verständnisses von Joyce' Texten führt. Die Sprache hat traumatischen Charakter für den Psychotiker. Worte ähneln Parasiten, Krebsen (Lacan 2005). Der kreative Akt des Schreibens vermittelt den Charakter einer Gegenwehr. Leser/-innen werden hineingezogen in ein spannungsreiches und vielfach unverständliches Ambiente. Obwohl die Signifikanten bekannt klingen, wird ihnen durch neologistische Verformung entlang homonymer Klanggebilde fremder Sinn zuteil.

Die „homonymische Reserve“ (Lacan 2006) von Klanggebilden lässt sich anhand des Titels des Seminar XXIII beispielhaft beschreiben. Was der Ausdruck Sinthom heißen kann, ist in seinem Klangbild vereinigt und bleibt darin verborgen. Erst die Schrift- 
form trifft eine Festlegung. Denn der Ausdruck Sinthom, ein von Lacan eingeführtes Wort, lässt sich gemäß dem Klang im Französischen als symptôme, als Symptom, als saint homme, heiliger Mann, aber auch als St. Thome, heiliger Thomas, oder saint tome, heiliger Band, anschreiben (Lacan 2005). Bei einer solchen Umformung werden entlang der Achse des Klangs Signifikanten gefunden, die auf andere, bekannte Signifikate verweisen. Auf diese Bildungsregel des Unbewussten stützt sich auch Freud, wenn er etwa die Espe und andere Signifikanten mit den Initialen des Wolfsmanns, S.P., in Verbindung bringt (Freud 1918).

Die mit einer Psychose verbundene kreative Leistungsfähigkeit stützt sich aber nicht nur auf bekannte Signifikanten, weshalb die Schrift, um die es bei Joyce geht, keine Schrift ist, die vom Signifikanten kommt (Lacan 2005). Sondern unter Ausnützung der homonymischen Reserve werden neue Sinnzusammenhänge erschlossen. Lacan selbst bediente sich über viele Jahre einer solchen Wortbildungstechnik (Benabou et al. 2002; Kadi 2012a), wovon das „sym, qui ptôme“ zeugt. In formaler Hinsicht ist auch Herrn S.' „Jah, preisen tut weh“(Stompe 2010) in dieser Weise gebildet. Aus dem Namen „Jahwe“, der nur durch die schriftliche Form der Falldarstellung als solcher zu erkennen ist (es bleibt in der Falldarstellung unklar, wie die Schreibweise mit dem Patienten geklärt wurde), ist durch Einfügung von bekannten Signifikanten ein Satz mit einem neuen Sinn kreiert worden.

Dieser Umgang mit der Schrift ist Ausdruck eines Genießens. Das gilt keineswegs generell für die Schrift. Im Gegenteil: Die Schrift, die vom Signifikanten kommt und insofern einen symbolischen Aspekt verkörpert, ist nicht Ausdruck einer Fülle, sondern vielmehr durchtränkt von einer Erfahrung des Mangels. Gegenüber dem Klang, dem gesprochenen und zunächst mütterlich konnotierten Wort vermittelt das Schreiben von Signifikanten eine Erfahrung der Kastration (Stockreiter 2012), weil - wie oben am Beispiel des Ausdrucks Sinthome beschrieben - mit der Verschriftlichung durch die Festlegung auf eine Schreibweise eine Reduktion von Sinn eintritt. Aber anders als solch ein Schreiben, welches sich auf mit anderen Sprecher/-innen geteilte Zeichen und Signifikanten zu stützen genötigt wird und insofern den Restriktionen des Symbolischen unterliegt, breitet sich das Schreiben des Psychotikers ins Reale aus. Aus neuen Schreibweisen sprossen neue Worte mit neuem Sinn. Diese Schrift vertritt Reste eines körperlichen, unstrukturierten und insofern entgrenzten Genießens.

Weder Herrn noch Frau S. war solch ein Schreiben konkret im Rahmen ihrer psychotischen Erfahrungen zugänglich. Frau S. berichtet vom Abschluss ihrer Diplomarbeit, von einem Schreiben also, das sich innerhalb symbolischer akademischer Restriktionen zu entfalten hatte. Im Unterschied zu Joyce' Schreiben wären diese schriftlichen Produktionen nicht als Sinthom anzusehen.

Mit der produktiven Schrift ist von Lacan freilich nicht nur der konkrete Schreibzusammenhang angesprochen, sondern auch der Prozess einer Produktion eines Sinthoms im Sinne eines Ego, das als vierter Ring den Fehler der Verknüpfung korrigiert. Lacan betont, dass dieses Ego nicht zu verwechseln sei mit dem Ich/moi der Spiegelbeziehung, welches der wahnhaften Wahrnehmung des eigenen Körpers entstammt. Dem sinthomatischen Ego fehlt der Bezug zum gestalteten Körper. Es entspricht vielmehr dem Lust-Ich des primären Narzissmus, das sich noch vor einer Scheidung zwischen Innen und Außen darbietet (Lacan 2005).

Die Zustände, in die Frau S. gerät, lassen sich mit den Epiphanien von Joyce vergleichen, in deren Rahmen das Unbewusste an das Reale geknüpft wird (Lacan 2005). 
Frau S. begreift sich in der floriden Phase ihrer Erfahrung als Jean-Paul Sartre und als Thomas Bernhard, wobei sie beide mit dem Schreiben in Verbindung bringt. Als Romy Schneider versetzt sie sich in Filmwelten, in denen sie nicht nur über ein weiteres, wahnhaftes Ego als Dirigent, sondern auch über die Aussicht auf den Nobelpreis für Philosophie, überreicht von Jacques Derrida, verfügt. Frau S. kommt mit diesen wahnhaften Bildungen in den Genuss von großen Lustprämien. Der Wegfall dieser Lustprämien, als sie ihr wahnhaftes Ego verloren hat, erklärt etwas von der depressiven Verstimmung, in die Frau S. in der Folge gerät.

\section{Literatur}

Apollon W (1991) Theory and practice in the psychoanalytic treatment of psychosis. In: Ragland-Sullivan E, Bracher M (Hrsg.) Lacan and the subject of language. 116-140. Routledge New York London

Apollon W (2002) The letter of the body. In: Apollon W, Bergeron D, Cantin L (Hrsg.) After Lacan. Clinical practice and the subject of the unconscious. 103-115. State University of New York Press New York

Aulagnier P (1975) La violence de l'interprétation - du pictogramme à l'énoncé. PUF Paris

Bénabou M, Cornaz L, de Liège D, Pélissier Y (2002) 789 Néologismes de Jacques Lacan. Epel Paris

Bergeron D (1990) L'enjeu de la cure du psychotique. In: Apollon W, Bergeron D, Cantin L (Hrsg.) Traiter la psychose. 139-159. Gifric Quebec

Brousse MH (2009) Ordinary psychosis in the light of Lacan's theory of discourse. Psychoanalytical Notebooks. The London Society of the New Lacanian School 19, 7-19

Castanet H (1994) Regard et perversion. Z'éditions Nice

Cremniter D, Maleval IC (1989) Contribution au diagnostic de psychose. Ornicar 48, 69-98

Fellahian C (2005) La psychose selon Lacan. Évolution d'un concept. l'Harmattan Paris

Fink B (1999) A clinical introduction to Lacanian psychoanalysis: theory and technique. University Press Harvard

Freud S (1894) Die Abwehr-Neuropsychosen: Versuch einer psychologischen Theorie der akquirierten Hysterie, vieler Phobien und Zwangsvorstellungen und gewisser halluzinatorischer Psychosen. In: Freud S GW I. 59-74. Fischer Frankfurt am Main

Freud S (1909) Der Familienroman der Neurotiker. In: Freud S GW VII. 227-231. Fischer Frankfurt am Main

Freud S (1911) Psychoanalytische Bemerkungen über einen autobiographisch beschriebenen Fall von Paranoia (Dementia paranoides). In: Freud S GW VIII. 240-316. Fischer Frankfurt am Main

Freud S (1917) Vorlesung: Die Libidotheorie und der Narzissmus. In: Freud S GW XI. 427-446. Fischer Frankfurt am Main

Freud S (1918) Nachträge aus der Urzeit - Lösung. In: Freud S GW XII. 122-137. Fischer Frankfurt am Main

Freud S (1922) „Psychoanalyse“ und "Libidotheorie“. In: Freud S GW XIII. 211-233. Fischer Frankfurt am Main

Freud S (1937) Der Mann Moses und die monotheistische Religion. In: Freud S GW XVI. 103-246. Fischer Frankfurt am Main

Kadi U (1999) Bilderwahn. Arbeit am Imaginären. turia + kant Wien

Kadi U (2002) Frauenmuster-Musterfrauen. RISS 53/1, 67-85

Kadi U (2006) Keine Frau, kein Körper. RISS 64/3, 87-111

Kadi U (2011) Subjektlos: den Anderen los? http://stuzzicadenti.at/2011-08-31/subjektlos/ (zugegriffen am 31.8.2011)

Kadi U (2012) Der wahnsinnige König. Zu Wahn und Verstehen bei Jaspers und Lacan. In: Unterthurner G, Kadi U (Hrsg.) Wahn. Philosophische, psychoanalytische und kulturwissenschaftliche Perspektiven. 87-106. turia + kant Wien

Kadi U (2012a) Bücherwurmeierspiel. Erscheint in: Waniek-Laquièze E, Berz P, Kubaczek M, Pias C, Unterholzner D (Hrsg.) Spielregeln. 20 Aufstellungen. Eine Festschrift für Wolfgang Pircher. diaphanes Berlin Zürich

Lacan I (1933) Le problème du style et la conception psychiatrique des formes paranoïaques de l'expérience. Minotaure 1, 68-69

Lacan I (1933a) Motifs du Crime Paranoïaques: Le Crime des Soeurs Papin. Minotaure 3-4, 25-28 
Lacan I (1973) Das Spiegelstadium als Bildner der Ichfunktion, wie sie uns in der psychoanalytischen Erfahrung erscheint. In: Lacan | Schriften I. 61-70. Walter Verlag Olten

Lacan I (1973a) L'étourdit. Scilicet 4, 5-25

Lacan I (1975) Über eine Frage, die jeder möglichen Behandlung der Psychose vorausgeht. In: Lacan I Schriften II. 61-117. Walter Verlag Olten

Lacan I (1975a) De la psychose paranoïaque dans ses rapports avec la personnalité. Seuil Paris

Lacan I (1975b) Die Bedeutung des Phallus. In: Lacan I Schriften II. 119-32. Walter Verlag Olten

Lacan I (1977) Ouverture de la section clinique. Ornicar 9, 7-14

Lacan I (1980) Vortrag über die psychische Kausalität. In: Lacan I Schriften III. 123-171. Walter Verlag Olten

Lacan I (1980a) Die Familie. In: Lacan I Schriften III. 39-100. Walter Verlag Olten

Lacan I (1986) Das Seminar. Buch XX (1972-1973). Encore. Quadriga Weinheim Berlin

Lacan I (1991) Subversion des Subjekts und Dialektik des Begehrens im Freudschen Unbewussten. In: Lacan I Schriften II. 165-204. 3. Aufl. Quadriga Weinheim Berlin

Lacan I (1997) Das Seminar. Buch III (1955-1956). Die Psychosen. Quadriga Weinheim Berlin

Lacan I (2005) Le Séminaire. Livre XXIII. Le sinthome. 1975-1976. Seuil Paris

Lacan I (2005a) Joyce le symptom. In: Lacan I Le Séminaire. Livre XXIII. Le sinthome. 1975-1976. 161-169. Seuil Paris

Lacan I (2006) Das Seminar. Buch V. Die Bildungen des Unbewußten. turia + kant Wien

Lacan I (o.J.) R.S.I. 1974-1975. Seminar XXII. Lacan Archiv Bregenz. Übersetzung für den vereinsinternen Gebrauch von Max Kleinert

Lacoue-Labarthe P, Nancy F-L (1973) Le titre de la lettre. Une lecture de Lacan. Galilée Paris

Lévy-Valensi I, Migault P, Lacan I (1931) Écrits „inspirés“: schizographie. Les Annales médico-psychologiques II, 508-522

Maleval I-C (2003) Elements pour une apprehension clinique de la psychose ordinaire. http://w3.erc.univ-tse2. $\mathrm{fr} /$ pdf/elements_psychose_ordinaire.pdf (zugegriffen am 23.7.2011)

Miller I-A (2005) La psychose ordinaire, Convention d'Antibes. Agalma Paris

Morel G (2000) Ambiguités sexuelles. Sexuation et psychose. Anthropos Paris

Morel, G (2007) Das Symptom, das Phantasma und die Pathologien des Gesetzes. RISS 65/1, 57-91

Morel $G$ (2008) La loi de la mère. Essai sur le sinthome sexuel. Anthropos Paris

Rank 0 (2007) Der Doppelgänger. Eine psychoanalytische Studie. Neuauflage der Schrift von 1925 mit einem Nachwort von Mladen Dolar. turia + kant Wien

Recalcati M (2008) Madness and Structure in Jacques Lacan. lacanian ink 32. 97-121

Roudinesco E (1993) Jacques Lacan. Esquisse d'un vie, histoire d'un système de pensée. Fayard Paris

Ruhs A (1995) Mangel/Geschlecht/Differenz. texte. psychoanalyse. ästhetik. kulturkritik. 15, 47-59

Ruhs A (2001) Das aufgebrochene Junktim: die „Psychoanalyse“ der Psychose. Betrachtungen aus der Sicht der strukturalen Psychoanalyse Lacans. In: Gondek, H-D, Hofmann R, Lohmann H-M. (Hrsg) Jacques Lacan Wege zu seinem Werk. 74-94. Klett-Cotta Stuttgart

Ruhs A (2005) Zur Problematik einer psychotherapeutisch relevanten Diagnostik mit besonderer Berücksichtigung der psychoanalytischen Perspektive. In: Bartuska H, Buchsbaumer M, Mehta G, Pawlowsky G, Wiesnagrotzky S (Hrsg.) Psychotherapeutische Diagnostik. Leitlinien für den neuen Standard. 147-153. Springer Wien New York

Skriabine P (2009) Ordinary psychosis with a Borromean approach. Psychoanalytical Notebooks. The London Society of the New Lacanian School 19, 45-55

Stockreiter K (2012) Das Reale der Schrift. In: Unterthurner G, Kadi U (Hrsg.) Wahn. Philosophische, psychoanalytische und kulturwissenschaftliche Perspektiven. 233-252. turia + kant Wien

Stompe T (2010) Ein Mordversuch als wahnhafte parasuizidale Handlung. JNNP 11, 1-3

Thibierge S (2003) Begehren. http://www.freud-lacan.com/Champs_specialises/Langues_etrangeres/Allemand/ Begehren (zugegriffen am 12.7.2011)

Waelhens De A, VerEcke W (2001) Phenomenology and Lacan on schizophrenia after the decade of the brain. University Press Leuven

Ver Ecke W (2009) Philosophical questions about the theory of psychosis in early Lacan. Psychoanalytical Notebooks. The London Society of the New Lacanian School 19, 233-239 